\title{
Increased $\alpha 1$-adrenoreceptor activity is required to sustain blood pressure in female rats under food restriction
}

\author{
Aline Arlindo de Souza, Rodrigo Cunha de Menezes*, Aline Rezende Abreu, Glaucy Rodrigues Araujo, \\ Daniela Caldeira Costa, Deoclecio Alves Chianca Jr
}

Department of Biological Sciences, Institute of Exact and Biological Sciences, Federal University of Ouro Preto (UFOP), Ouro Preto, MG 35400-000, Brazil

\section{A R T I C L E I N F O}

\section{Article history:}

Received 3 September 2014

Accepted 11 February 2015

Available online 2 March 2015

\section{Keywords:}

Food restriction

$\alpha 1$-Adrenoreceptors

Cardiovascular reflexes

\begin{abstract}
A B S T R A C T
Aims: We evaluated the effect of food restriction (FR) on the various reflexes involved in short term cardiovascular regulation; we also evaluated the contribution of the sympathetic nervous system and of the plasmatic nitric oxide (NO) in the development of the counterregulatory cardiovascular changes triggered by FR.

Main methods: Female rats were subjected to FR for 14 days, and after this period biochemical measurements of biochemical parameters were performed. For physiological tests, animals were anaesthetised, and a catheter was inserted into the femoral artery and vein for the acquisition of blood pressure and heart hate, and drug infusion, respectively. We then tested the Bezold-Jarisch reflex, the baroreflex and chemoreflex and the effect of the infusion of adrenergic receptor antagonists in control and food restricted animals.

Key findings: The rats subjected to severe FR presented biochemical changes characteristic of malnutrition with a great catabolic state. FR also led to hypotension and bradycardia besides reducing the plasmatic concentration of NO. Moreover, activation of the Bezold-Jarisch reflex induced a more pronounced hypotensive response in animals subjected to FR. Intravenous infusion of a $\alpha 1$-adrenoreceptor antagonist induced a greater hypotensive response and a more pronounced tachycardic response in animals under food restriction, while the infusion of $\beta$ adrenoreceptor antagonist induced lower increases in blood pressure in these animals.

Significance: Our results suggest that an increased $\alpha 1$-adrenoreceptor activity in the resistance arteries coupled with a reduction of plasmatic NO contributes in a complementary manner to maintain the blood pressure levels in animals under FR.
\end{abstract}

(c) 2015 Elsevier Inc. All rights reserved.

\section{Introduction}

Food restriction (FR) model has been widely used as an animal model of the nutritional aspect of anorexia nervosa [37], simulating the reduced food intake observed in this condition. Studies using this model have demonstrated that a dietary restriction can trigger the development of cardiovascular diseases such as ventricular hypertrophy and hypotension [39].

Studies have shown an adjustment in cardiovascular function after a period of reduced food intake [1,3]. The main cardiovascular changes in severe dietary restriction are hypotension and bradycardia. McKnight et al. $[29,30]$, observed that rats submitted to a $25 \%$ reduction in daily food intake for 14 days became bradycardic and hypotensive [29,30]. In another study, a severe restriction, a reduction of forty percent (40\%), for 8 weeks, in spontaneously hypertensive rats, induced a

\footnotetext{
* Corresponding author at: Universidade Federal de Ouro Preto (UFOP), Instituto de Ciencias Exatas e Biologicas, Departamento de Ciencias Biologicas, Laboratorio de Fisiologia Cardiovascular, Ouro Preto, MG 35400-000, Brazil. Tel.: + 55313559 1224; fax: + 553135591680 .

E-mail address: rodrigo.menezes@iceb.ufop.br (R.C. de Menezes).
}

reduction in blood pressure [32]. Furthermore, in humans, reduction in food intake is very common among patients with anorexia nervosa who also present bradycardia and hypotension [15].

Other cardiovascular disorders such as electrocardiographic abnormalities, left ventricular hypertrophy, systolic dysfunction, ventricular remodelling, diastolic myocardial dysfunction, decreased cardiac contraction and decrease of lateral ventricle relaxation $[10,35]$ are also related to severe reduction in food intake.

Considering that severe acute FR can cause cardiovascular diseases, the regulatory mechanisms involved in the regulation of the cardiovascular function could be compromised, leading to the pathological conditions cited above. Remarkably, other dietary challenges can also disrupt the cardiovascular regulatory mechanisms. In this regard, several studies have shown that post-weaning protein malnutrition, in rats, leads to changes in key systems involved in the regulation of systemic blood pressure [5]. Protein malnutrition causes an increased sensibility of the baroreceptor reflex, the chemoreflex [34] and the Bezold-Jarisch [40], a reflex from the heart and lungs that, when activated, leads the inhibition of sympathetic activity [4].

Moreover, rats fed a low protein diet present an increased sympathetic tone to the heart, indicating that the autonomic nervous system 
is compromised in these rats [28]. Furthermore, Tropia and colleagues [40] have shown that protein restricted rats present an increased vascular sympathetic tone [40]. Another study observed endothelial dysfunction with increases in superoxide anion and nitric oxide in these animals [13].

Therefore, knowing that FR may cause cardiovascular abnormalities, and that dietary challenges can modulate the mechanisms responsible for cardiovascular control, we hypothesized that there might be adjustments in these regulatory systems, which may be essential to maintain the cardiovascular homeostasis in rats subject to FR. Thus, to test this hypothesis we evaluated the effect of FR on baroreflex, Bezold-Jarisch reflex and chemoreflex. We have also evaluated the contribution of the adrenergic receptors and of the plasmatic nitric oxide in the development of the counterregulatory cardiovascular changes triggered by severe FR.

\section{Methods}

\section{Ethical approval}

All procedures were approved by the ethics committee for animal research of the Federal University of Ouro Preto (CEUA-UFOP; no. 2010/35), and were performed according to the regulations set forth by the UK Animals (Scientific Procedures) Act 1986, the Guidelines for Reporting Animal Research [20], Directive 2010/63/EU and by the National Institutes of Health Guidelines for the Care and Use of Laboratory Animals (NIH publication no. 85-23, revised 2010) and according to the journal policies and regulations on animal experimentation. All efforts were made to minimize the number of animals used in the study, and to avoid any unnecessary distress to the animals.

\section{Animals}

All experiments were conducted on female Fischer rats $(n=70)$ at the Federal University of Ouro Preto (Brazil). We chose female rats because the anorexia is more common in woman. Animals were acquired from the University Centre of Animal Sciences, and were maintained in a dark-light cycle of $12 \mathrm{~h}$ with the room temperature maintained at $24{ }^{\circ} \mathrm{C}$. The rats weighted between 200 and $220 \mathrm{~g}$, and were housed in cages individually and separated in two groups: a control group $(\mathrm{n}=$ 34 ) or food restricted group $(n=36)$. Both groups were fed a commercial chow (Nuvilab, Brazil) as appropriated. Free access to water was allowed. The animal's oestrous cycle was evaluated every day.

\section{Diet protocol}

To assess dietary intake, the control animals weighting between 200 and $220 \mathrm{~g}$ were placed in individual metabolic cages. We evaluated the food intake and the animal's weight at 12:00 pm daily. The average food consumption was determined for these animals after 14 days. This average was used to set the amount of food to be offered to animals that were to be submitted to food restriction. The food-restricted animals were fed $40 \%$ of the control group average consumption. Animals under food restriction remained in individual boxes and had their weight measure every day, always before offering food, which was performed once a day for 14 days. On the 14th day control or restriction animals were subjected to the experimental protocols. All animals received the same diet, Nuvilab ${ }^{\circledR}$, only varying the amount of food offered. Daily, after measuring the animal's weight, we have also evaluated the oestrous cycle. For that, vaginal secretion was collected with a plastic pipette filled with $10 \mu \mathrm{L}$ of normal saline $(\mathrm{NaCl} 0.9 \%)$ by inserting the tip into the rat vagina. Vaginal fluid was placed on glass slides. A different glass slide was used for each cage of animals. One drop was collected with a clean tip from each rat. Unstained material was observed under a light microscope, without the use of the condenser lens, with 10 and $40 \times$ objective lenses. Proestrous consists of a predominance of nucleated epithelial cells; oestrous primarily consists of enucleated cornified cells; metoestrus consists of the same proportion among leukocytes, cornified, and nucleated epithelial cells, and dioestrus smear primarily consists of a predominance of leukocytes [26].

Characterization of the effects of food restriction on the animal's biochemical profile

To evaluate the effect of food restriction on metabolic profiles and their similarity with anorexia nervosa we used an exclusive group, without surgical procedures, for collecting blood samples and performing biochemical measurements. At the end of the $14 \mathrm{~d}$ of restricted $(n=8)$ or control diet $(n=8)$, animals were fasted for $12 h$, then they were anaesthetised with isoflurane, and blood was collected through the brachial plexus. All samples were centrifuged at $13,000 \mathrm{~g}$ for $10 \mathrm{~min}$ to separate serum from plasma, and were stored in sterilized tubes at $-80{ }^{\circ} \mathrm{C}$ according to future use. For measurements using plasma, we previously added to sterilized tube anticoagulant GLISTAB KF 12 g/dL 6 g/dL + EDTA (LABTEST Diagnostica, MG, Brazil) in the amount of 1 drop/3 mL blood. However, when serum is used, blood was stored in sterilized tube without anticoagulant. The biochemical tests were made as described in the protocols of Labtest Kits (Labtest Diagnostic, MG, Brazil) [38]. We quantified urea, creatinine, haemoglobin, albumin, alkaline phosphatase, total protein, glucose, ALT, AST, triglycerides, total cholesterol, HDL cholesterol and LDL cholesterol in both groups. The dosage of NO was taken by Griess method through colorimetric quantification of the nitrite using the BioAssay system kit (DINO - 250).

\section{Surgical procedures}

Fourteen days after the commencement of the diet treatments, the animals were subjected to catheter implantation, as described previously [25]. Briefly, rats were anaesthetised (2.5\% isoflurane in $3 \mathrm{~L} / \mathrm{min}_{2}$; Cristalia, Brazil) and polyethylene catheters were inserted into the femoral artery (for cardiovascular measurements) and into the femoral vein (for drug infusion) of all animals. The catheters were tunnelled subcutaneously and exteriorized at the back of the neck. After surgery, analgesics (ketoflex $4 \mathrm{mg} / \mathrm{kg}, 0.1 \mathrm{~mL} / 300$ g s.c., Mundo Animal, Brazil) and antibiotics $(0.2 \mathrm{~mL} / 100 \mathrm{~g}$, s.c., Fort Dodge Animal Health, Brazil) were administered. The animals were maintained in individual cages in order to recover from the anaesthesia. Experimental procedures began $48 \mathrm{~h}$ after the procedure.

\section{Cardiovascular measurements}

The arterial catheter was connected to a pressure transducer MLT0699 (ADI Instruments, Australia), which was connected to a signal amplifier ETH-400 (CB Sciences Inc., USA). The analogical signal from the amplifier was digitized by a 12 bit analogical-to-digital converter (PowerLab/400, ADI Instruments, Australia), and the pulsatile arterial pressure recorded at $1000 \mathrm{~Hz}$ by the software Chart 7.0 for Windows (ADInstruments, Australia). MAP and HR were derived on-line from the pulsatile arterial pressure using pulse-to-pulse analysis [16].

\section{Experimental design}

Experiments were performed, as follows, in a room in which the temperature was maintained at $24-25^{\circ} \mathrm{C}$. On the day of the experiment, animals were brought to the experimental room, in their home cages, $2 \mathrm{~h}$ prior to the beginning of the protocol. The experiment commenced only after stabilization of physiological parameters, such as heart rate and mean arterial pressure (HR and MAP) for at least $30 \mathrm{~min}$.

The first experiment evaluated the influence of food restriction on cardiovascular reflexes. The evaluation of baroreflex, in animals fed ad libitum $(\mathrm{n}=10)$ and animals that were food restricted $(\mathrm{n}=10)$ was 
made by infusing, intravenously, $0.1 \mathrm{~mL}$ of phenylephrine $(299 \mathrm{nM} / \mathrm{mL}$, infusion velocity: $2.2 \mathrm{~mL} / \mathrm{h}$ ), sodium nitroprusside $(335.6 \mathrm{nM} / \mathrm{mL}$, infusion velocity: $1.5 \mathrm{~mL} / \mathrm{h}$ ) or vehicle in the same volume, using a $5 \mathrm{~mL}$ Hamilton syringe $(57 \mathrm{~mm}$ ) mounted in an infusion pump (Insight, SP, Brazil). After the cardiovascular parameters returned to baseline levels, the chemoreflex was evaluated ( $C n=9$ vs. FR $n=7)$. For that, potassium cyanide (KCN: $15.3 \mu \mathrm{M} / \mathrm{kg}-0.1 \mathrm{~mL}$ ) was administered (i.v.) in bolus. Approximately 15 min after the infusion of KCN, the BezoldJarisch reflex was evaluated by infusing phenylbiguanide (23.4 nM/ $\mathrm{kg}-0.1 \mathrm{~mL}$ ) in bolus ( $\mathrm{C}=9 \mathrm{vs}$. FR $\mathrm{n}=7$ ).

The second series of experiments evaluated the influence of food restriction on the $\alpha 1$ - and $\beta$-adrenergic receptor activities on the resting cardiovascular parameters, in two separate sets of groups. In the first group, alpha-adrenergic activity was evaluated by analysing the effects of a bolus injection of prazosin hydrochloride (i.v.; $2.3 \mu \mathrm{M} / \mathrm{Kg}$ ), an $\alpha 1$ receptor antagonist on baseline MAP and HR in control $(n=10)$ and food restricted animals $(n=10)$. In the second group, $\beta$-adrenergic activity was evaluated by analysing the effect of a bolus injection of propranolol (i.v.; $33.8 \mu \mathrm{M} / \mathrm{Kg}$ ), a non-selective beta-adrenergic receptor antagonist on baseline MAP and HR in control $(n=6)$ and food restricted animals $(\mathrm{n}=8)$.

\section{Data analysis}

Data for HR and MAP values were recorded continuously. The baseline values for MAP and HR were obtained by averaging the values of the 5 min-period that preceded drug injections. Maximal changes (as means \pm standard error of the mean) were calculated using 3 min averages or the peak response (30 s) after the drug infusion. In infusion ramp (baroreflex test), the data (MAP and HR) from each experimental group were organized in a two-column table with data ordered from the lowest to the highest MAP, along with the respective HR values.
These data were divided into $10 \mathrm{~mm} \mathrm{Hg}$ pressure bins. For each pressure bin, one MAP \pm SEM value was plotted against its respective $\mathrm{HR} \pm \mathrm{SEM}$ value. The baroreflex curve was then fitted to a sigmoidal logistic equation:

$H R=H R \max +\frac{H R \min -H R \max }{1+e^{-\left(\frac{\text { MAP }-M A P 50}{b}\right)}}$

where HRmin is the lower plateau, HRmax is the upper plateau, $b$ is the curvature coefficient, and MAP50 is the MAP at the midpoint of the HR range. Individual sigmoidal functions were averaged in order to determine the mean sigmoidal fit for the group. The derivatives of individual sigmoidal baroreflex curves were calculated and averaged within an experimental group in order to determine the baroreflex gain-coefficient curve. The maximal changes in MAP and HR were evaluated and the gain coefficient was calculated by the equation:

Gain $=\frac{\triangle \max H R}{\triangle \operatorname{maxMAP}}$

where $\triangle$ maxMAP is the maximal change in MAP after injection of sodium nitroprusside and $\triangle \operatorname{maxHR}$ is the maximal change in HR due to the changes in MAP caused by the pharmacological manoeuvre [7].

\section{Statistical analysis}

Prism 5.0 (GraphPad Software, La Jolla, CA, USA) was used to analyse all data. The data are expressed as mean \pm standard error of the mean (SEM). Student's paired t-test was used to analyse the differences within groups. Student's unpaired t-test or two way (diet and time/weight as factors) analysis of variance (ANOVA) in comparison with Bonferroni post-test was used to analyse differences between groups. Mann-
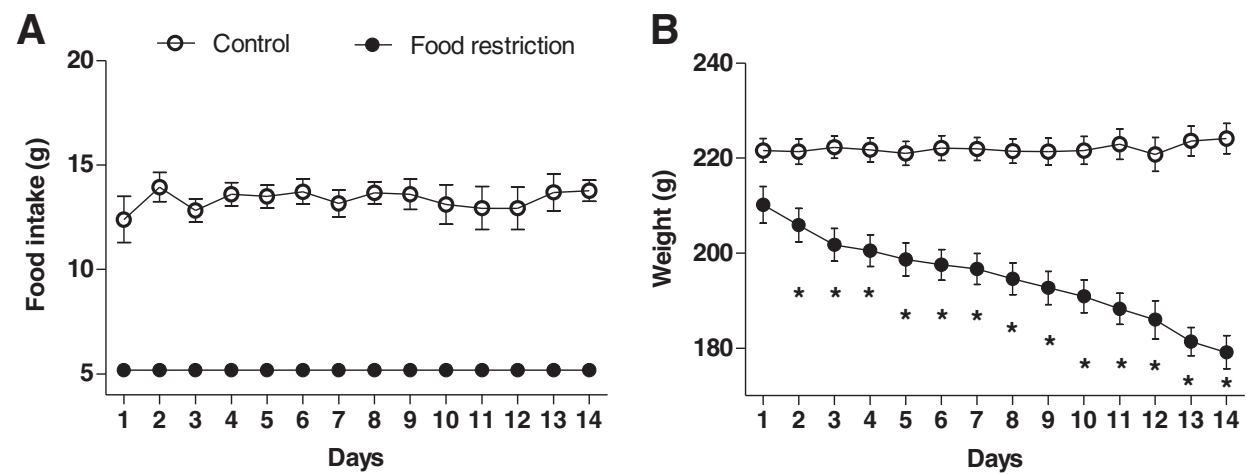

C

D
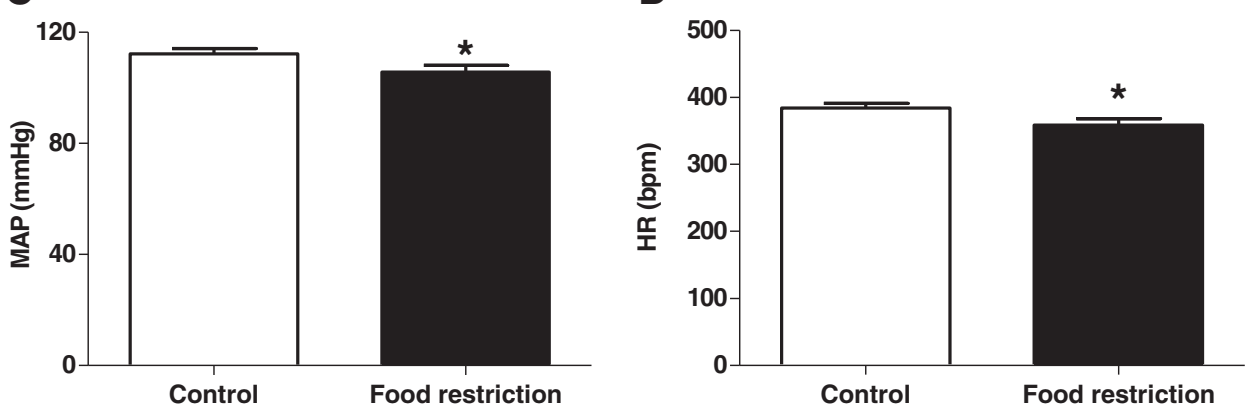

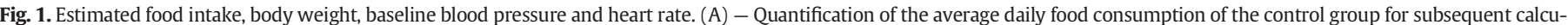

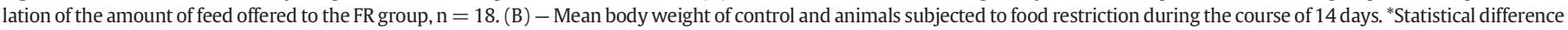

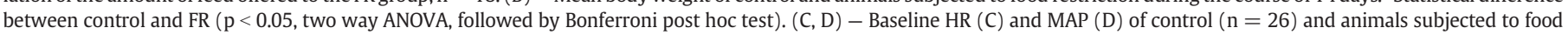
restriction $(\mathrm{n}=28)$. *Statistical difference between control and $\mathrm{FR}(\mathrm{p}<0.05$, Student's unpaired t-test). 
Table 1

Effect of control and food restriction (60\%) diets for 14 days in female rats. Separate columns should be used for measures of variance (SD, SE etc.), the \pm sign should not be used.

\begin{tabular}{|c|c|c|c|c|c|}
\hline $\begin{array}{l}\text { Biochemical } \\
\text { parameter }\end{array}$ & C & $\mathrm{SEM} / \mathrm{max}-\mathrm{min}$ & FR & $\mathrm{SEM} / \mathrm{max}-\mathrm{min}$ & $\mathrm{p}$ value \\
\hline Urea (mg/dL) & 44.95 & 0.99 & 58.56 & 3.14 & $0.0010^{*}$ \\
\hline Creatinine (mg/dL) & 1.21 & 0.03 & 1.03 & 0.04 & $0.0040^{*}$ \\
\hline Haemoglobin (g/dL) & 14.88 & 1.14 & 12.42 & 0.88 & 0.1103 \\
\hline Albumin $(\mathrm{g} / \mathrm{dL})$ & 2.83 & 0.04 & 2.39 & 0.06 & $<0.0001^{*}$ \\
\hline Alkaline phosphatase (U/L) & 42.99 & 1.73 & 47.12 & 1.90 & 0.1302 \\
\hline Total protein $(\mathrm{g} / \mathrm{dL})$ & 6.98 & 0.208 & 6.73 & 0.11 & 0.3199 \\
\hline Glucose $(\mathrm{mg} / \mathrm{dL})$ & 213.0 & $(173.6-258.4)$ & 258.9 & $(203.9-432.6)$ & $0.0208^{*}$ \\
\hline ALT & 35.02 & (31.31-44.99) & 28.18 & $(20.76-51.24)$ & 0.3181 \\
\hline AST & 77.71 & 1.51 & 72.76 & 1.45 & $0.0336^{*}$ \\
\hline Triglycerides (mg/dL) & 37.92 & 3.68 & 29.40 & 2.17 & 0.0662 \\
\hline Total cholesterol (mg/dL) & 96.92 & 7.09 & 69.22 & 3.08 & $0.0030^{*}$ \\
\hline HDL cholesterol (mg/dL) & 43.20 & 5.14 & 39.10 & 3.09 & 0.5062 \\
\hline LDL cholesterol (mg/dL) & 53.73 & 9.54 & 30.12 & 3.92 & $0.0382^{*}$ \\
\hline
\end{tabular}

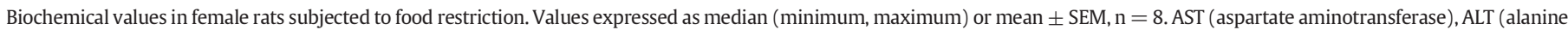

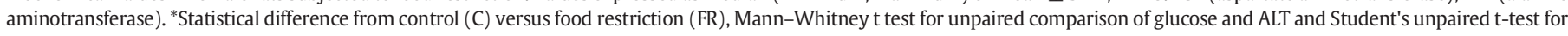
other parameters $(\mathrm{p}<0.05)$.

Whitney t test was used for nonparametric comparisons. The significance threshold level was set at 0.05 .

\section{Results}

Food restriction induced weight loss, hypotension bradycardia and changes in biochemical parameters

The rats were divided into control (C) and food restriction groups (FR). The daily average of food consumed by the control group was $13.50 \mathrm{~g} \pm 0.2 \mathrm{~g}$ or $158.15 \mathrm{~kJ}(\mathrm{n}=18)$. Thus the amount of food offered daily to the FR group was $5.5 \mathrm{~g}$ or $63.17 \mathrm{~kJ}(\mathrm{n}=18)$ (Fig. 1A). The animals submitted to food restriction showed a progressive reduction in body weight from the second day until the end of the diet protocol, with the final body weight in the restricted animals been approximately 15\% less than the control animals (Fig. 1B) (Final weight - C: $224.1 \mathrm{~g} \pm$ 3 vs. FR: $179.2 \mathrm{~g} \pm 3, \mathrm{p}<0.001)$. Animals fed a restricted diet presented a significantly lower baseline MAP (C: $112.3 \mathrm{~mm} \mathrm{Hg} \pm 1.8, \mathrm{n}=26$ vs. FR: $105.6 \mathrm{~mm} \mathrm{Hg} \pm 2.5, \mathrm{n}=28 ; \mathrm{p}=0.04$, by Student's unpaired t-test) and HR (C: 384.5 bpm $\pm 6.8, \mathrm{n}=26$ vs. FR: 359 bpm $\pm 9.4, \mathrm{n}=28 ; \mathrm{p}=$ 0.0336 , by Student's unpaired t-test) in comparison with controls (Fig. 1 C and D).

Along with the evaluation of the body weight and quantification of food intake, we evaluated the daily oestrous cycle setting how many days each animal remained in each phase. The animals in the control diet showed a normal oestrous cycle, alternating between ovulatory and not ovulatory cycles. However, the animals under FR, from the second week of restriction and on, did not ovulate anymore, staying only in the metoestrus and dioestrus cycles (data not shown). There were significant decreases in the plasma concentrations of creatinine, albumin, aspartate aminotransferase (AST), total cholesterol and LDL cholesterol in the FR group when compared to the controls (Table 1). On the other

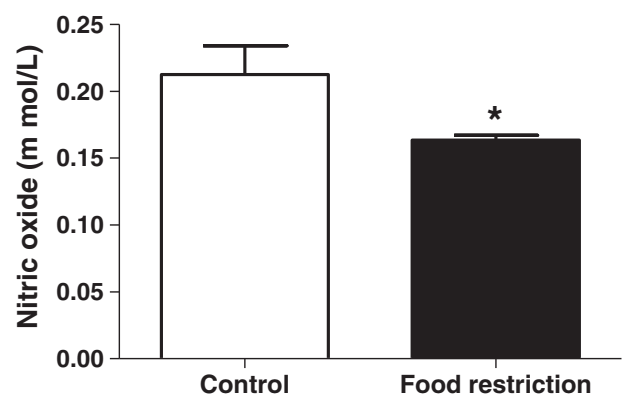

Fig. 2. Effects of food restriction on the nitric oxide system. Determination of plasmatic nitric oxide $(n=7)$. Effect of food restriction on plasma nitric oxide. Statistical difference between control and FR; Student's t-test unpaired $(\mathrm{p}<0.05)$. hand, urea and glucose plasma levels increased in FR as shown in Table 1.

\section{Food restriction reduces plasma nitric oxide levels}

To examine the influence of FR on the nitric oxide system, we measure the plasmatic NO concentration. The plasma nitric oxide concentration was decreased in the FR group when compared to the control group $(\mathrm{p}=0.0437$, by Student's unpaired t-test) (Fig. 2).

Food restriction potentiates the Bezold-Jarisch reflex but does not alter the chemoreflex and the baroreflex activity

In order to evaluate the influence of food restriction on cardiovascular reflexes, we examine the activity of three reflexes: the BezoldJarisch reflex, the chemoreflex and the baroreflex.

The injection of phenylbiguanide produced the Bezold-Jarisch reflex in both groups, however, it induced a more pronounced hypotensive response in animals subjected to food restriction ( $p=0.0070$, by Student's unpaired t-test) (Fig. 3C). The bradycardic response was similar in both groups (Fig. 3D).

The intravenous infusion of potassium cyanide for chemoreflex evaluation generated similar increases in MAP in both groups $(p=0.1815$, by Student's unpaired t-test; Fig. 3A). It also induced similar decreases in HR ( $p=0.1185$, by Student's unpaired t-test; Fig. 3B). The baroreceptor reflex was measured by a ramp infusion of sodium nitroprusside and phenylephrine intravenously. To evaluate the baroreflex, the function curve was divided into the following variables: lower plateau, upper plateau, MAP50, gain and heart rate range (Table 2). There were no significant differences in these parameters. The graphic representation of the baroreflex function curve and the grouped representation of the gain are presented on Fig. 4A and B, respectively.

\section{Food restriction increases $\alpha 1$-adrenergic receptor activity}

To evaluate the FR influence on the adrenergic receptor activity, we examined the effect of the infusion of $\alpha 1$ - and $\beta$-adrenergic receptor antagonists on resting MAP and HR. After the intravenous bolus of prazosin, an $\alpha 1$-adrenergic receptor antagonist, we observed a greater hypotensive response in animals under food restriction $(p=0.0225$, by Student's unpaired t-test) and consequently a more pronounced tachycardic response in the same group ( $\mathrm{p}=0.0188$, by Student's unpaired t-test) (Fig. 5). The infusion of propranolol, a $\beta$-adrenergic antagonist, induced a comparable bradycardia in both groups $(p=0.4981$, by Student's unpaired t-test). On the other hand, the blockade of $\beta$ receptors produced significantly lower increases in MAP in animals subjected to food restriction ( $\mathrm{p}=0.0003$, by Student's unpaired t-test) (Fig. 6). 


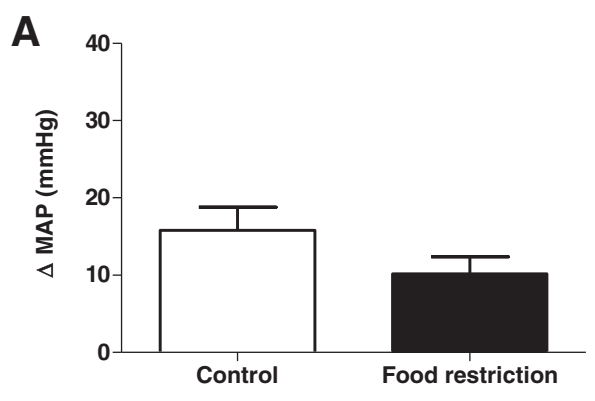

C

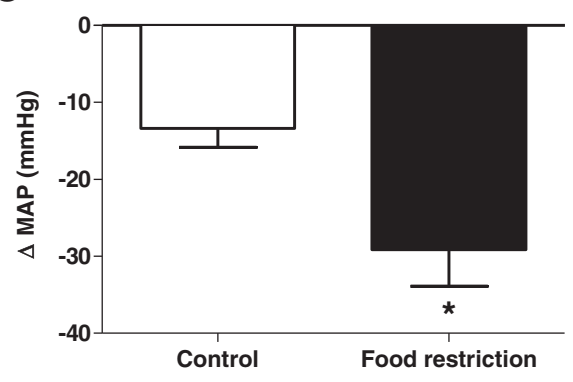

E Control
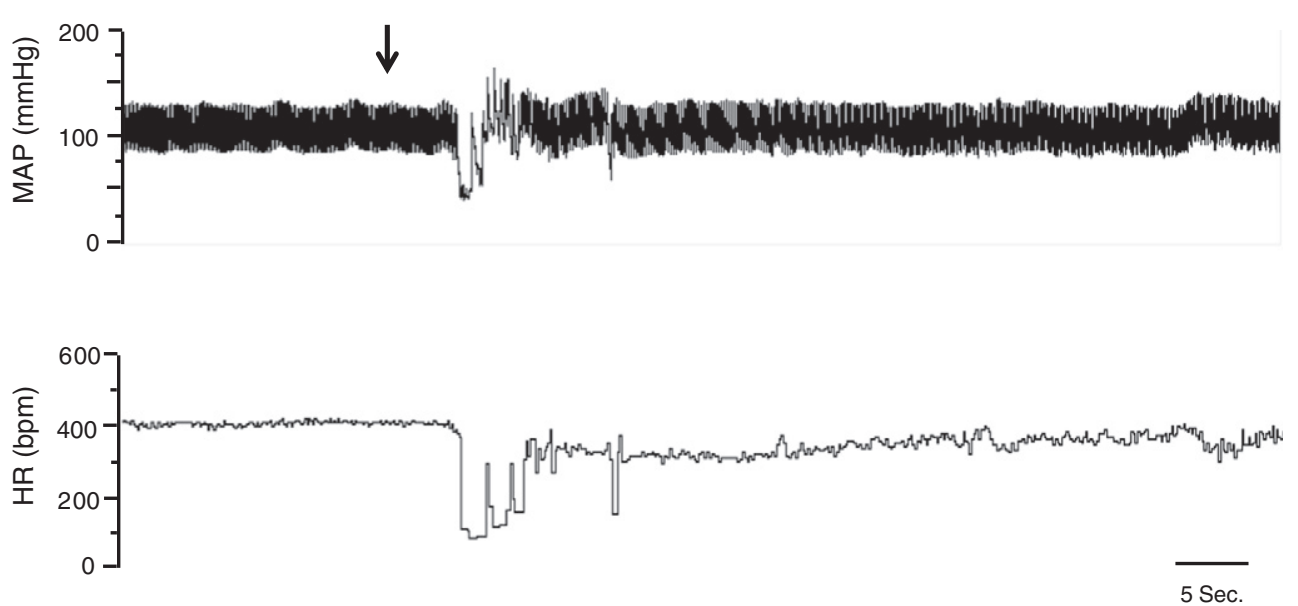

F $\quad$ Food restriction
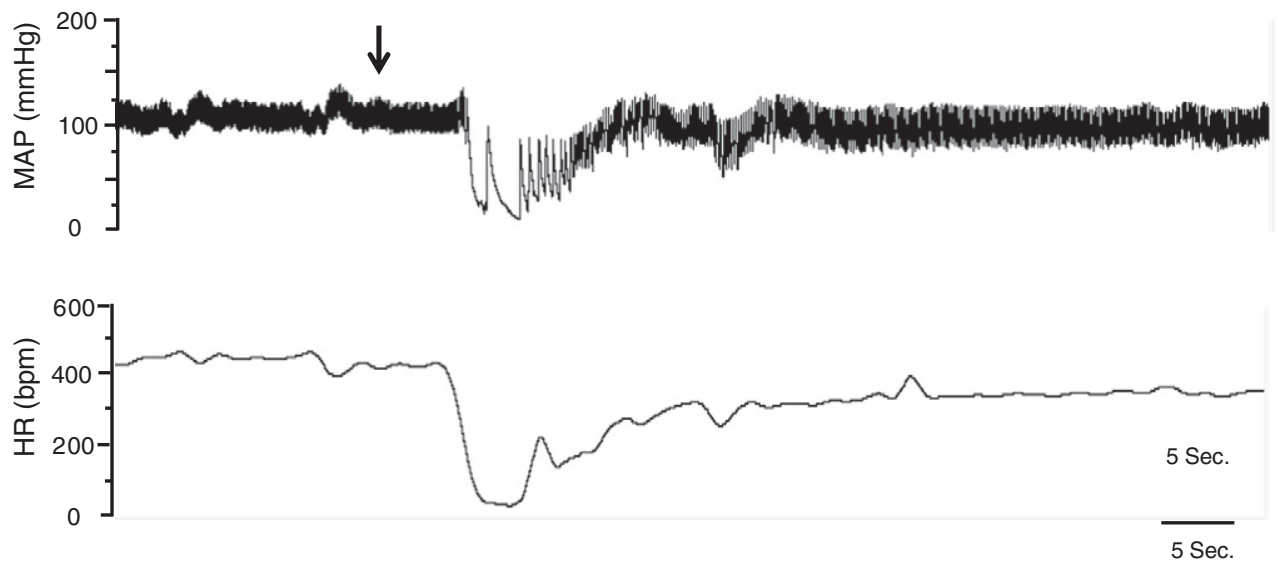

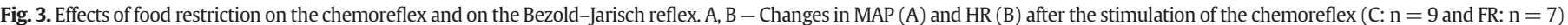

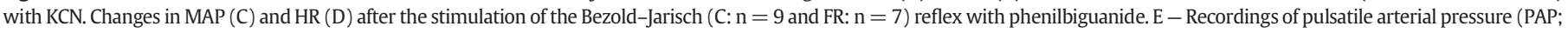

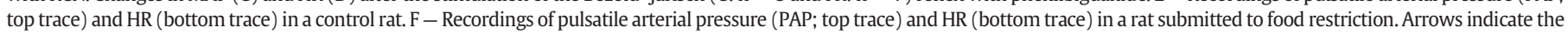
infusion of phenylbiguanide. *Statistical difference between control and FR; Student's unpaired t-test $(\mathrm{p}<0.05)$. 
Table 2

Baroreflex curve parameters of control and food restriction rats.

\begin{tabular}{lllll}
\hline Parameter & Control & SEM & Food restriction & SEM \\
\hline Upper plateau $(\mathrm{bpm})$ & 465.9 & 20.84 & 434.9 & 15.26 \\
Lower plateau $(\mathrm{bpm})$ & 300.9 & 15.64 & 286.2 & 25.61 \\
Heart rate range $(\mathrm{bpm})$ & 165.0 & 29.40 & 185.3 & 40.67 \\
MAP50 $(\mathrm{mm} \mathrm{Hg})$ & 115.6 & 5.20 & 112.6 & 5.15 \\
Gain $(\mathrm{bpm} / \mathrm{mm} \mathrm{Hg})$ & -8.38 & 1.34 & -8.71 & 1.56 \\
\hline
\end{tabular}

Data are reported as means \pm SEM for 10 rats in each group. MAP50 = mean arterial pressure at the midpoint of the heart rate range. Student t-test.

\section{Discussion}

In this study we evaluated the effect of food restriction on the various systems involved in cardiovascular regulation. We showed that the $\alpha 1$-adrenergic receptor activity is increased in animals under FR. We have also found that the hypotensive component of the BezoldJarisch reflex is more pronounced in these animals. Moreover, these animals presented a reduced plasma nitric oxide concentration. Our data indicates that an increased responsiveness of $\alpha 1$-adrenergic receptors, in arteries, coupled with a reduced concentration of the vasodilator agent nitric oxide, appears to be essential for the maintenance of the blood pressure levels in animals submitted to food restriction.

We used a model food restriction with $60 \%$ reduction in the quantity of calories and nutrients offered for 14 days. After this period we observed that animals fed this restricted diet lost approximately $15 \%$ of their body weight, which is very similar to what was observed in studies that have used this deprivation protocol $[8,27]$. Although the animals under food restriction presented a significant weight loss, they did not show any signs of sickness.

In our experiments, we have used female rats because anorexia is more prevalent in women, then, our model of food restriction is appropriate, since it simulates the nutritional state of anorexia. However, it is well known that certain cardiovascular disorders occur with different incidences between male and female, as a consequence of the presence or absence of the estradiol, such as hypertension, pulmonary hypertension, changes in the angiotensin system or in oxidative stress $[2,11,22]$. We cannot discard the possibility that the changes observed in the animals submitted to food restriction could be, partly, caused by the changes in the oestrous cycles observed in these animals. As it is cited above, FR animals, from the second week of food restriction and onwards, stopped ovulating which could have led to a reduction of the plasmatic estradiol. Several studies have reported that the reduction, or absence, of estradiol in females could lead to cardiovascular disorders [14,23, 41], thus if the FR animals have a reduction of estradiol, even for a week, this could lead to some of the changes in the cardiovascular control that we have observed. However, it is important to point out that, even though it is well known that the animal's behaviour changes between the oestrous phases, there are no reports showing that it can also influence the cardiovascular system.

Rats submitted to severe food restriction presented alterations in biochemical parameters related to energy metabolism. These animals showed significant decreases in plasma creatinine, albumin, AST, total cholesterol and LDL cholesterol. However, glucose and urea plasma concentration increased after food restriction. These data indicate that rats under food restriction developed malnutrition, which can be demonstrated by the decreased albumin concentration and increased concentration of plasmatic urea, suggesting a high protein catabolism. Our results corroborate previous data which showed that people who consume hypocaloric diets develop hypoalbuminemia [43], increased plasmatic urea and decreased creatinine clearance [42]. We also show that these animals develop marked changes in lipid metabolism, which can be demonstrated by a reduction of total cholesterol and LDL. Interestingly, anorexic patients, whom also have a restricted diet, also present decreased total cholesterol and LDL [9]. One factor contributing to the increase of plasmatic glycaemia could be the likely increase in the production of cortisol. This rise in circulating cortisol is common in severe restricted diets [31], and is related to the stress that the hypocaloric diets generate [39]. The increase in corticosterone during the food restriction might be essential to these animals' lives, since corticosterone facilitates the conversion of amino acids into glucose (i.e. gluconeogenesis), which would avoid the hypoglycaemia derived from the reduced food consumption, and thus protecting the brain. Notably the protocol of food restriction used in our experiments induced similar biochemical changes to the ones found in patients with anorexia [9].

In the present study we have also observed that animals under food restriction presented a significantly lower baseline MAP and HR, corroborating previous data from different research groups, both in rats and in humans $[18,35]$. However, the foundation of these changes can be multifactorial since hypovolemia [6], cardiac dysfunctions [10] or dysfunctions in the reflexes that control the blood pressure (BP) [19] could lead to both bradycardia and hypotension. Considering the fact that food restriction, and for that matter anorexia in human beings, alters the cardiovascular system causing hypotension and bradycardia, we designed protocols to investigate few systems involved in blood pressure regulation.

We observed a decreased plasmatic concentration of NO, a signalling molecule, with a powerful vasodilator effect [12], in animals fed the restricted diet. Given that dietary restriction causes hypotension in these animals, it can be suggested that the reduction in plasmatic nitric oxide could occur as a compensatory reaction to the decreased blood pressure, which would cause less shear in the vessel diminishing the stimulus for NO production [21], and thus preventing an excessive reduction of BP.

It is well known that reflexes in the body control blood pressure moment to moment, and changes in the activity of these reflexes could lead
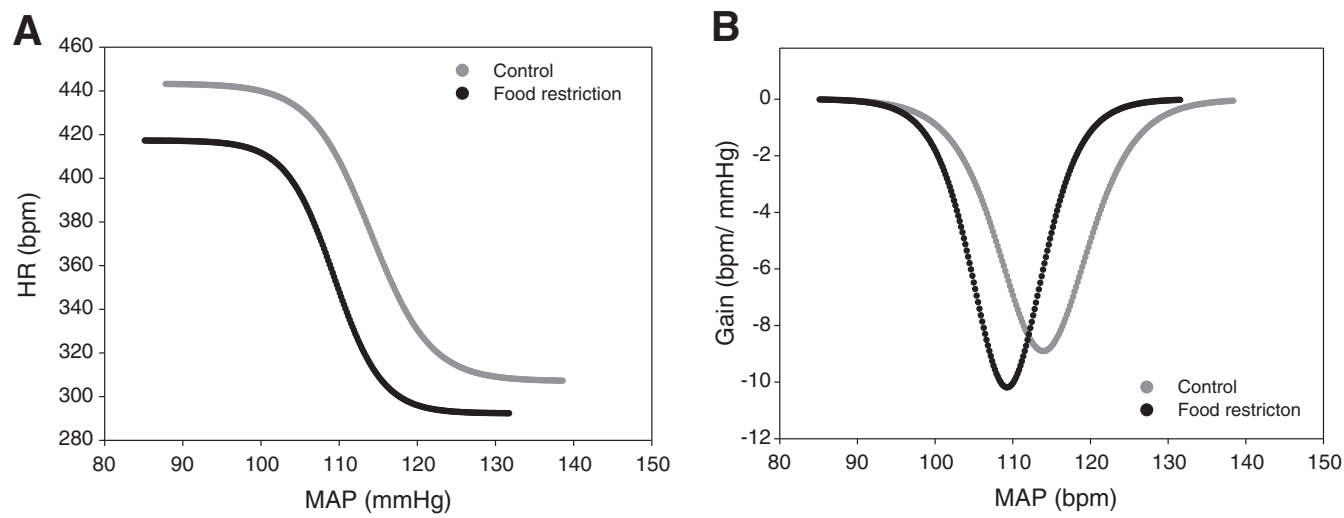

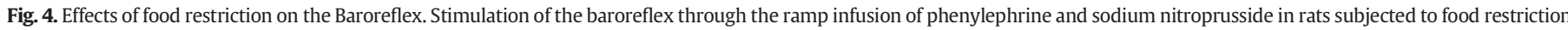

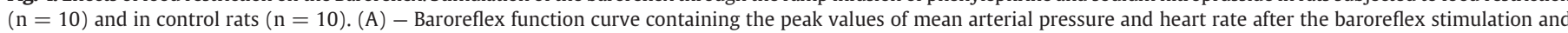
(B) baroreflex gain, i.e., the first derivative of the sigmoid functions at a specific mean arterial pressure values. 
A

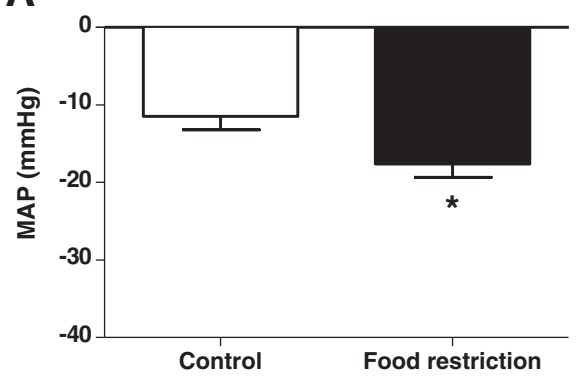

B

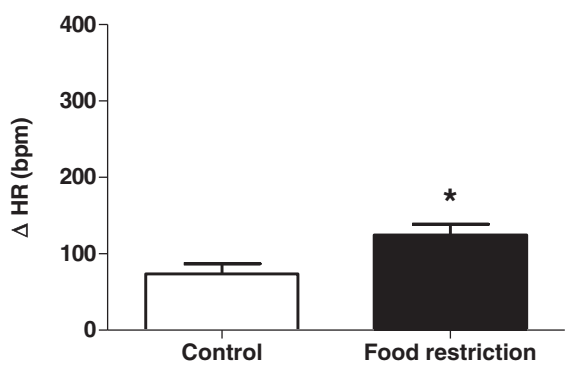

C Control
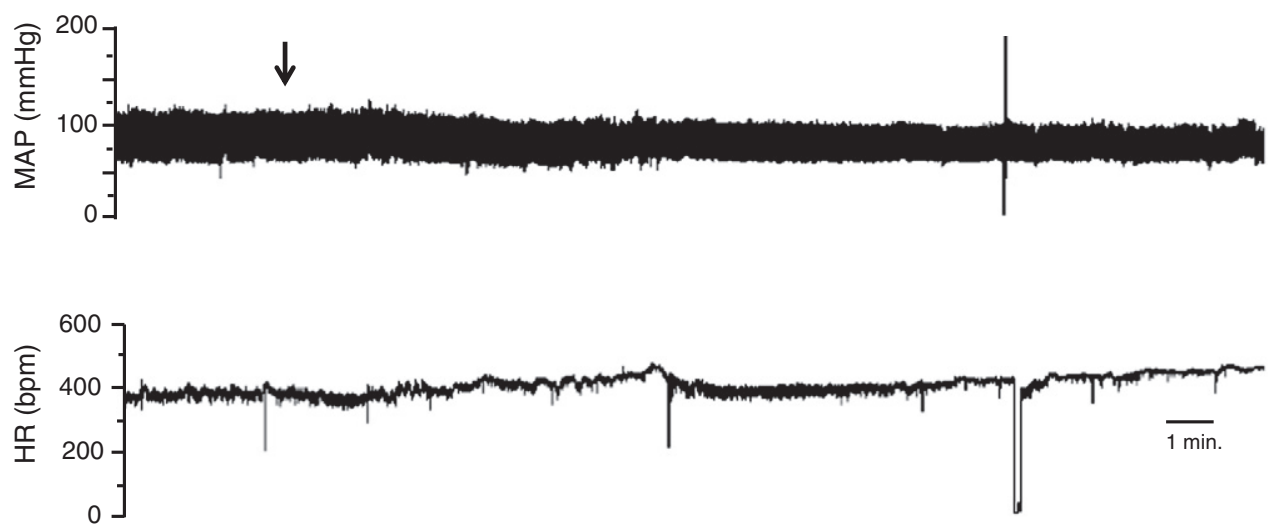

D

Food restriction
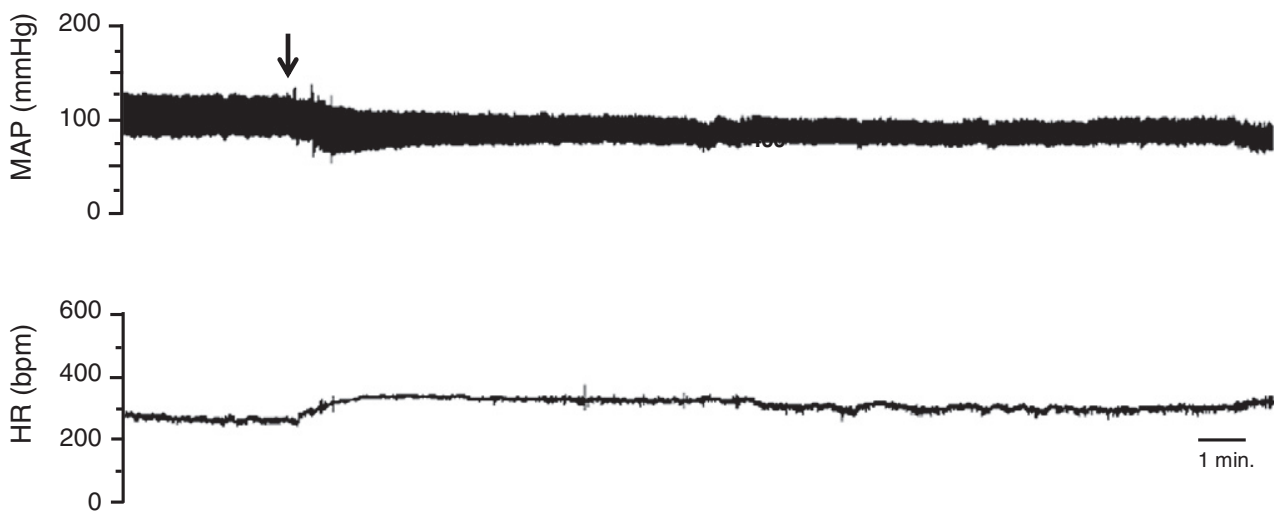

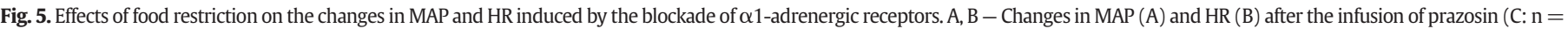

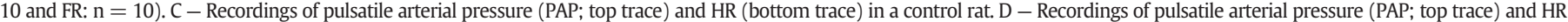
(bottom trace) in a rat submitted to food restriction. Arrows indicate the infusion of prazosin. *Statistical difference between control and FR; Student's unpaired t-test ( $\mathrm{p}<0.05$ ).

to changes in BP levels $[17,33]$. Animal studies have shown that absence of a balanced diet (i.e. low protein diets) can affect the sensitivity of these reflexes [5,34]. In our study, we showed that the Bezold-Jarisch reflex is compromised in rats under severe food restriction. The activation leads to sympathetic inhibition causing hypotension and bradycardia [24]. The food restricted animals showed an increased hypotensive response to the injection of phenylbiguanide, suggesting that food restriction leads to a likely increase of the $\alpha 1$-adrenergic receptor activity in the arteries. The chemoreflex activity was not affected in animals under food restriction. Surprisingly, the baroreflex sensitivity was also not affected by food restriction. Our result contradicts those presented in the literature, where patients under severe diet restrictions presented an increase in baroreflex gain $[18,19]$. This disparity may be explained by differences between the protocols utilized in our study and in the previous studies. In our report, we used pharmacological manoeuvres, to test the baroreflex, which cannot be accomplished in humans, and the techniques used in humans are made by indirect means, such as spectral analysis or by analysis of postural changes. Considering that the activity of adrenergic receptors could be altered by food restriction, we designed experiments to evaluate the activity of $\alpha 1$ - and $\beta$ adrenoreceptor activities, indirectly, in this paradigm.

In order to pursue this answer, we infused, intravenously, prazosin and propranolol, and $\alpha 1$ - and $\beta$-adrenoreceptor antagonists, respectively. The infusion of prazosin induced hypotension and tachycardia in both groups; nevertheless, these responses were more pronounced in the animals under a severe food restriction. Possible explanations for this increase are augmented receptor sensitivity or density, or both, or even increases in endogenous ligand release from sympathetic 

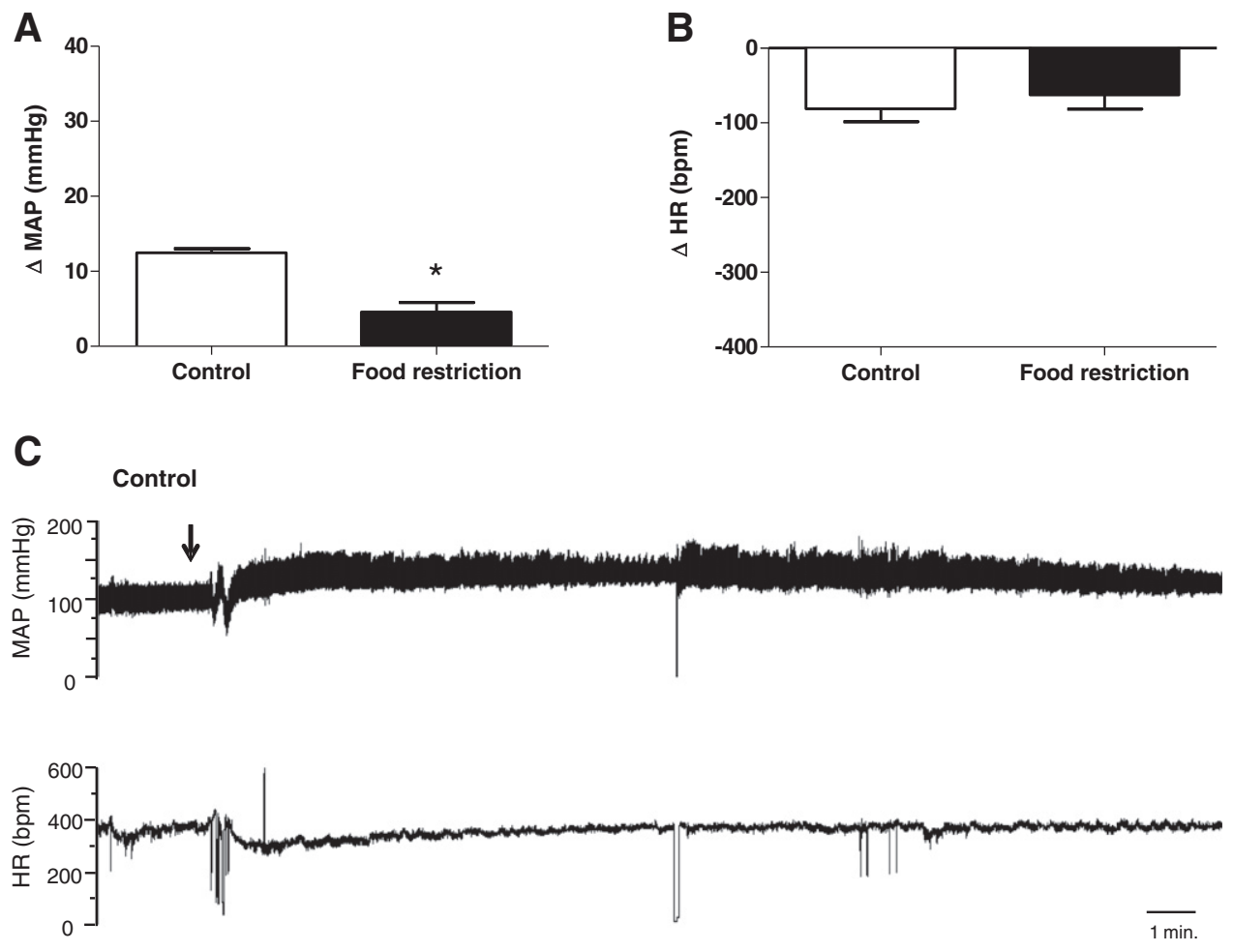

D
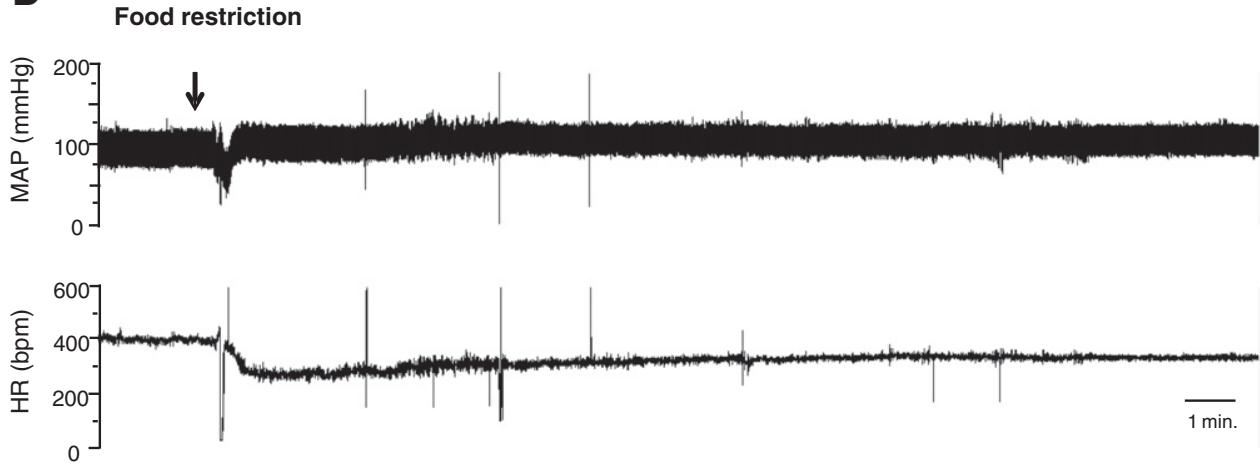

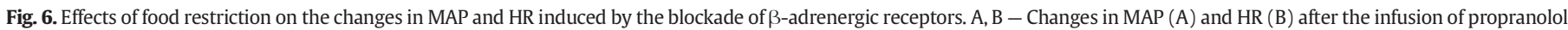

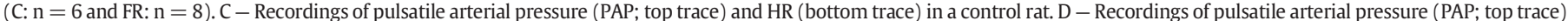

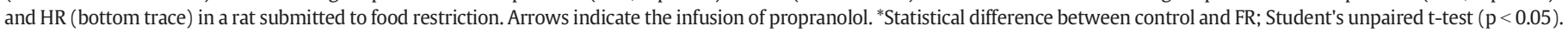

terminals in the blood vessels. These results indicate an increased $\alpha 1$ adrenergic receptor activity in these animals, with only 14 days of food restriction, which would be necessary to sustain the blood pressure. Importantly, animals fed a low protein diet, for 35 days, present a higher vasomotor tone that could be due to an increased sympathetic efferent activity $[16,40]$, corroborating the idea that dietary restrictions can lead to increased SNS activation to the arteries. The pronounced tachycardic response in these animals could be, possibly, the result of the pronounced hypotension, which would activate the baroreflex circuit leading to a marked activation of the SNS to the heart.

It is important to notice that in our experiments FR leads to hypoalbuminemia, which could cause hypovolemia. Importantly, a previous study, using a longer period of food restriction, showed that FR leads to a reduction in haematocrit and consequently in blood volume [36]. Our model uses a shorter restriction period than the study cited above, thus if there is a hypovolemic state it is probably much milder. Even if this reduction in blood volume is mild, it may be the cause of the activation of the Bezold-Jarisch reflex as well as in the sympathetic nervous system. This reduction in volume could lead to the reduction in BP observed in our study. Therefore, the increased activity of $\alpha 1$-adrenergic receptors could be, partly, due to this decrease in BP, and act as a counterregulatory measure to those changes, and could be essential to the maintenance of "normal" BP levels, which are necessary to survival.

In keeping with this investigation of the role of adrenoreceptors in cardiovascular changes observed in animals submitted to food restriction, we have also evaluated the effect of the blockade of the $\beta$ adrenergic receptors by infusing propranolol. The infusion of propranolol induced a significant bradycardia in both groups, showing that the response of beta-receptor in the heart appears to be normal in animals under food restriction. Nevertheless, the raise in MAP, that accompanied the referred bradycardia, was blunted in the animals under food restriction. The hypertension observed after the infusion of propranolol is probably due to a baroreflex response, triggered by the bradycardia, causing a compensatory increase in BP. This response occurs through the activation of $\alpha 1$-adrenergic receptors in the resistance arteries, which appear to be hyper activated in animals under FR, as shown previously. The inability to stimulate a receptor that is, possibly, at its saturation threshold, may be the limiting factor for the blood response in FR rats, justifying the smaller change in MAP in these animals after the propranolol infusion. 


\section{Conclusions}

In conclusion, our results suggest that food restriction leads to a hypotensive state that is counteracted, largely, by an increased activity of $\alpha 1$-adrenoreceptors in the resistance arteries. Importantly, the concentration of NO, a vasodilator agent, is low in these animals, suggesting that the NO system is also involved in avoiding a more pronounced hypotensive state. Thus, the present study shows that these two systems contribute in a complementary manner to support the blood pressure levels in animals under food restriction allowing the cardiovascular system to maintain sufficient blood supply to the tissues.

\section{Conflict of interest statement}

The authors declare that there are no conflicts of interest.

\section{Author contributions}

Experiments were performed at the Cardiovascular Physiology Lab and at the Biochemistry and Molecular Biology Lab, Department of Biological Sciences, Federal University of Ouro Preto, Brazil. Conception and design of the experiments: A.M.A.S., R.C.D.M., D.A.C.J.; collection, analysis and interpretation of data: A.M.A.S., G.R.A., A.R.R.A., D.C.C., R.C.A.M.; drafting and revising the article critically for important intellectual content: A.M.A.S., R.C.D.M. All authors approved the final version of the manuscript.

\section{Funding}

This work is supported by the Fundacao de Amparo a Pesquisa do Estado de Minas Gerais (FAPEMIG) (APQ-01543-14), Programa de Apoio a Nucleos Emergentes (PRONEM) (APQ-04721-10), Coordenacao de Aperfeiçoamento de Pessoal de nivel Superior (CAPES), Conselho Nacional de Desenvolvimento Cientifico e Tecnologico (CNPq) and Universidade Federal de Ouro Preto (UFOP).

\section{References}

[1] P.B. Alden, R.D. Madoff, T.J. Stahl, D.J. Lakatua, W.S. Ring, F.B. Cerra, Left ventricular function in malnutrition, Am. J. Physiol 253 (2 Pt 2) (1987) H380-H387.

[2] E.D. Austin, T. Lahm, J. West, S.P. Tofovic, A.K. Johansen, M.R. Maclean, A. Alzoubi, M. Oka, Gender, sex hormones and pulmonary hypertension, Pulm. Circ. 3 (2) (2013) 294-314.

[3] V. Balasubramanian, N.S. Dhalla, Biochemical basis of heart function. V. Effect of starvation on storage, transport, and synthesis of cardiac norepinephrine in rats, Can. J. Physiol. Pharmacol. 50 (3) (1972) 238-243.

[4] L.B. Bell, K.P. O'hagan, P.S. Clifford, Cardiac but not pulmonary receptors mediate depressor response to IV phenyl biguanide in conscious rabbits, Am. J. Physiol 264 (6 Pt 2) (1993) R1050-R1057.

[5] V.M. Bezerra, C.H. Xavier, R.C. De Menezes, M.A. Fontes, L.M. Cardoso, L.G. Fernandes, D.A. Chianca Jr., Bezold-Jarisch reflex in sino-aortic denervated malnourished rats, Auton. Neurosci. 162 (1-2) (2011) 48-53.

[6] A. Bouquegneau, B.E. Dubois, J.M. Krzesinski, P. Delanaye, Anorexia nervosa and the kidney, Am. J. Kidney Dis. 60 (2) (2012) 299-307.

[7] L.M. Cardoso, M.L. Pedrosa, M.E. Silva, M.F. Moraes, E. Colombari, D.A. Chianca Jr., Baroreflex function in conscious rats submitted to iron overload, Braz. J. Med. Biol. Res. 38 (2) (2005) 205-214.

[8] K.D. Carr, T.H. Park, Y. Zhang, E.A. Stone, Neuroanatomical patterns of Fos-like immunoreactivity induced by naltrexone in food-restricted and ad libitum fed rats, Brain Res. 779 (1-2) (1998) 26-32.

[9] T. Case, S. Lemieux, S.H. Kennedy, G.F. Lewis, Elevated plasma lipids in patients with binge eating disorders are found only in those who are anorexic, Int. J. Eat. Disord. 25 (2) (1999) 187-193.

[10] D. Casiero, W.H. Frishman, Cardiovascular complications of eating disorders, Cardiol. Rev. 14 (5) (2006) 227-231.

[11] S.H. Chou, Y.C. Lee, C.F. Huang, Y.R. Wang, H.P. Yu, Y.T. Lau, Gender-specific effects of caloric restriction on the balance of vascular nitric oxide and superoxide radical, Cardiovasc. Res. 87 (4) (2010) 751-759.

[12] S. Das, K.N. Kumar, Nitric oxide: its identity and role in blood pressure control, Life Sci. 57 (17) (1995) 1547-1556.

[13] A.C. De Belchior, J.K. Angeli, T.O. Faria, F.D. Siman, E.A. Silveira, E.F. Meira, C.P. Da Costa, D.V. Vassallo, A.S. Padilha, Post-weaning protein malnutrition increases blood pressure and induces endothelial dysfunctions in rats, PLoS One 7 (4) (2012) e34876.
[14] D.P. Dias, M. Oliveira, H.C. Salgado, R. Fazan Jr., Ovariectomy does not affect the cardiac sympathovagal balance of female SHR but estradiol does, Braz. J. Med. Biol. Res. 43 (10) (2010) 969-975.

[15] F. Galetta, F. Franzoni, A. Cupisti, D. Belliti, F. Prattichizzo, M. Rolla, QT interval dispersion in young women with anorexia nervosa, J. Pediatr. 140 (4) (2002) 456-460.

[16] J.M. Gomide, R.C. De Menezes, L.G. Fernandes, F.C. Silva, L.M. Cardoso, P.H. Miranda, L.G. Da Silva, M.P. Lima, J.L. Pesquero, G. Foureaux, A.J. Ferreira, D.A. Chianca Jr., Increased activity of the renin-angiotensin and sympathetic nervous systems is required for regulation of the blood pressure in rats fed a low-protein diet, Exp. Physiol. 98 (1) (2013) 57-66.

[17] G.A. Head, Baroreflexes and cardiovascular regulation in hypertension, J. Cardiovasc. Pharmacol. 26 (Suppl. 2) (1995) S7-16.

[18] T. Ishizawa, K. Yoshiuchi, Y. Takimoto, Y. Yamamoto, A. Akabayashi, Heart rate and blood pressure variability and baroreflex sensitivity in patients with anorexia nervosa, Psychosom. Med. 70 (6) (2008) 695-700.

[19] F. Jacoangeli, F.S. Mezzasalma, G. Canto, F. Jacoangeli, C. Colica, De L.A., F. Iellamo, Baroreflex sensitivity and heart rate variability are enhanced in patients with anorexia nervosa, Int. J. Cardiol. 162 (3) (2013) 263-264.

[20] C. Kilkenny, W.J. Browne, I.C. Cuthill, M. Emerson, D.G. Altman, Improving bioscience research reporting: the ARRIVE guidelines for reporting animal research, J. Pharmacol. Pharmacother. 1 (2) (2010) 94-99.

[21] G.K. Kolluru, S. Sinha, S. Majumder, A. Muley, J.H. Siamwala, R. Gupta, S. Chatterjee, Shear stress promotes nitric oxide production in endothelial cells by sub-cellular delocalization of eNOS: a basis for shear stress mediated angiogenesis, Nitric Oxide 22 (4) (2010) 304-315.

[22] K. Komukai, S. Mochizuki, M. Yoshimura, Gender and the renin-angiotensinaldosterone system, Fundam. Clin. Pharmacol. 24 (6) (2010) 687-698.

[23] Z. Liu, Y. Gou, H. Zhang, H. Zuo, H. Zhang, Z. Liu, D. Yao, Estradiol improves cardiovascular function through up-regulation of SOD2 on vascular wall, Redox Biol. 3 (2014) 88-99.

[24] J.C. Longhurst, Cardiac receptors: their function in health and disease, Prog. Cardiovasc. Dis. 27 (3) (1984) 201-222.

[25] I.O. Loss, L.G. Fernandes, C.D. Martins, L.M. Cardoso, M.E. Silva, V.J. Dias-da-Silva, M.F. Moraes, D.A. Chianca Jr., Baroreflex dysfunction in rats submitted to protein restriction, Life Sci. 81 (11) (2007) 944-950.

[26] F.K. Marcondes, F.J. Bianchi, A.P. Tanno, Determination of the estrous cycle phases of rats: some helpful considerations, Braz. J. Biol. 62 (4A) (2002) 609-614.

[27] P. Marinkovic, V. Pesic, N. Loncarevic, K. Smiljanic, S. Kanazir, S. Ruzdijic, Behavioral and biochemical effects of various food-restriction regimens in the rats, Physiol. Behav. 92 (3) (2007) 492-499.

[28] C.D. Martins, D.A. Chianca Jr., L.G. Fernandes, Cardiac autonomic balance in rats submitted to protein restriction after weaning, Clin. Exp. Pharmacol. Physiol. 38 (2) (2011) 89-93.

[29] K.A. Mcknight, H. Rupp, R.E. Beamish, N.S. Dhalla, Modification of catecholamineinduced changes in heart function by food restriction in rats, Cardiovasc. Drugs Ther. 10 (Suppl. 1) (1996) 239-246.

[30] K.A. Mcknight, H. Rupp, K.S. Dhalla, R.E. Beamish, N.S. Dhalla, Biphasic changes in heart performance with food restriction in rats, J. Appl. Physiol. 87 (5) (1999) 1909-1913.

[31] A. Oskis, C. Loveday, F. Hucklebridge, L. Thorn, A. Clow, Diurnal patterns of salivary cortisol and DHEA in adolescent anorexia nervosa, Stress 15 (6) (2012) 601-607.

[32] J.M. Overton, J.M. Vanness, R.M. Casto, Food restriction reduces sympathetic support of blood pressure in spontaneously hypertensive rats, J. Nutr. 127 (4) (1997) 655-660.

[33] J.F. Paton, L. Ratcliffe, D. Hering, J. Wolf, P.A. Sobotka, K. Narkiewicz, Revelations about carotid body function through its pathological role in resistant hypertension, Curr. Hypertens. Rep. 15 (4) (2013) 273-280.

[34] A.R. Penitente, L.G. Fernandes, L.M. Cardoso, M.E. Silva, M.L. Pedrosa, A.L. Silva, A.S. Haibara, M.F. Moraes, D.A. Chianca, Malnutrition enhances cardiovascular responses to chemoreflex activation in awake rats, Life Sci. 81 (7) (2007) 609-614.

[35] M.F. Pinotti, A.S. Leopoldo, M.D. Silva, M.M. Sugizaki, A.F. Do Nascimento, A.P. LimaLeopoldo, F.F. Aragon, C.R. Padovani, A.C. Cicogna, A comparative study of myocardial function and morphology during fasting/refeeding and food restriction in rats, Cardiovasc. Pathol. 19 (5) (2010) e175-e182.

[36] E. Schwartz, J.A. Tornaben, G.C. Boxill, The effects of food restriction on hematology, clinical chemistry and pathology in the albino rat, Toxicol. Appl. Pharmacol. 25 (4) (1973) 515-524

[37] Z. Siegfried, E.M. Berry, S. Hao, Y. Avraham, Animal models in the investigation of anorexia, Physiol. Behav. 79 (1) (2003) 39-45.

[38] M. Silva, L.F. Bonomo, R.P. Oliveira, L.W. Geraldo DE, M.E. Silva, M.L. Pedrosa, Effects of the interaction of diabetes and iron supplementation on hepatic and pancreatic tissues, oxidative stress markers, and liver peroxisome proliferator-activated receptor-alpha expression, J. Clin. Biochem. Nutr. 49 (2) (2011) 102-108.

[39] A.J. Tomiyama, T. Mann, D. Vinas, J.M. Hunger, J. Dejager, S.E. Taylor, Low calorie dieting increases cortisol, Psychosom. Med. 72 (4) (2010) 357-364.

[40] F.C. Tropia, L.M. Cardoso, M.L. Pedrosa, M.E. Silva, A.S. Haibara, M.F. Moraes, D.A. Chianca Jr., Effects of low-protein diet on the baroreflex and Bezold-Jarisch reflex in conscious rats, Nutr. Neurosci. 4 (2) (2001) 99-107.

[41] S. Turdi, A.F. Huff, J. Pang, E.Y. He, X. Chen, S. Wang, Y. Chen, Y. Zhang, J. Ren, 17-beta estradiol attenuates ovariectomy-induced changes in cardiomyocyte contractile function via activation of AMP-activated protein kinase, Toxicol. Lett. 232 (1) (2014) 253-262.

[42] A.P. Winston, The clinical biochemistry of anorexia nervosa, Ann. Clin. Biochem. 49 (Pt 2) (2012) 132-143.

[43] S.H. Yap, J.C. Hafkenscheid, J.H. Van Tongeren, Important role of tryptophan on albumin synthesis in patients suffering from anorexia nervosa and hypoalbuminemia, Am. J. Clin. Nutr. 28 (12) (1975) 1356-1363. 